\title{
MICRODIETAS NA ALIMENTAÇÃO DA TILAPIA DO NILO DURANTE A FASE DE REVERSÃO SEXUAL
}

\author{
HONORATO, Claucia Aparecida ${ }^{1}$ \\ TESSER, Marcelo Borges ${ }^{2}$ \\ PORTELLA, Maria Célia ${ }^{3}$ \\ CARNEIRO, Dalton José ${ }^{4}$
}

\begin{abstract}
RESUMO: O objetivo deste trabalho foi avaliar o uso de diferentes microdietas para fase de reversão sexual de tilápia do Nilo (Oreochromis niloticus). Foram utilizadas 4000 larvas de tilápia com 8,31 $\pm 0,14 \mathrm{~mm}$ de comprimento total. $\mathrm{O}$ experimento foi conduzido em delineamento inteiramente casualizado, com cinco tratamentos e quatro repetições. Os tratamentos utilizados foram: FSH-Farelada sem hormônio (controle); e as dietas com hormônio masculinizante (60 mg de 17- $\alpha$-metiltestosterona) FCH - Farelada; MAH - Microaglutinada; SSE microencapsulada spray dried sem material encapsulante; SCE - microencapsulada spray dried, 66,7\% de material encapsulante e 33,3\% de dieta. Foram realizadas biometrias no-início, aos 15 dias e ao final dos 30 dias de experimento. As larvas alimentadas com a dieta SSE apresentaram as melhores médias de peso e comprimento total, fator de condição e taxa de reversão sexual. O tratamento SCE demonstrou o menor desempenho sem comprometer o índice de reversão sexual. O tratamento com a dieta SSE resultou em um lote de peixe com maior uniformidade ao final de período de reversão sexual. Conclui-se que as microdietas MAH e SSE são as mais eficazes a serem utilizadas na fase de reversão sexual.
\end{abstract}

Palavra-chave: Microcápsulas. Processamento de microdietas. 17- $\alpha$-metiltestosterona

\section{MICRO DIET UTILIZATION FOR NILE TILAPIA Oreochromis niloticus LARVAL SEXUAL REVERSION}

SUMMARY: The objective of this work was to evaluate different microdiets for Nile tilapia (Oreochromis niloticus) sexual reversion phase. They were used 4000 tilapias larvae with $8.31 \pm 0,14 \mathrm{~mm}$ total length. The experiment was conducted with five treatments and four repetitions as followed, FSH - powder diet without hormone (control); and diets with hormone (60 mg of $17 \alpha$ methyl testosterone) FCH - powder diet, MAH Microaglomerated diet, SSE - spray dried microencapsulated diet without wall material, SCE - spray dried microencapsulated diet with wall material (66.7\% wall material and $33.3 \%$ of formulated diet). Weighing samples were conducted at the initiation of the experiment, at the $15^{\text {th }}$ and at the $30^{\text {th }}$ days. Larvae fed with SSE diet showed better weight and length means, condition factor sexual reversion ratio. On the other hand, treatment SCE showed worst growth performance with no compromise on sexual reversion ratio. The SSE resulted in better fish uniformity at the end of the sexual reversion phase. It can be concluded that the microdiets MAH and SSE were efficient and can be utilized for tilapia sexual reversion.

Keywords: Microencapsulated. Processing diets. $17 \alpha$ methyl testosterone.

\section{INTRODUÇÃO}

A criação de tilápia do Nilo (Oreochromis niloticus) é bastante difundida em países de clima tropical e subtropical devido ao seu alto valor comercial. Contudo, é indispensável para a sua produção

\footnotetext{
${ }^{1}$ Centro Universitário da Grande Dourados (UNIGRAN), Faculdade de Ciências Biológicas e da Saúde, Hospital Veterinário, Rua Balbina de Matos, 2121 - Jd. Universitário CEP 79.824-900 - Dourados/MS, clauciahonorato@yahoo.com.br

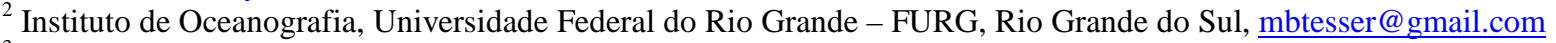

${ }^{3}$ Universidade Estadual Paulista, Departamento de Biologia Aplicada a Agropecuária, Unesp / Jaboticabal, São Paulo, portella@caunesp.unesp.br

Universidade Estadual Paulista (UNESP)/Centro de Aquicultura (CAUNESP), Jaboticabal, São Paulo, daltoncj@ caunesp.unesp.br
} 
racional a obtenção de populações masculinas, que apresentam taxas de crescimento superior às fêmeas, evitando problemas provenientes dos gastos energéticos com a desova, além do excesso populacional nos viveiros e desta espécie (MEURER et al., 2003).

A técnica que tem demonstrado melhor viabilidade para produção de populações monossexo de tilápia é a incorporação do hormônio masculinizante em dietas fareladas para as larvas (BEARDMORE $e t$ al., 2001). O hormônio mais comumente utilizado é o $17-\alpha$-metiltestosterona é incorporado à dieta na quantidade de 30 a 60 mg.kg-1 de ração (MAINARDES-PINTO et al., 2000). Autores descrevem que o tratamento tem início com larvas entre 9 a $11 \mathrm{~mm}$ de comprimento total, com duração de 21 a 30 dias, resultando em uma população de fêmeas inferior a 5\% (GREEN; TEICHERT-CODDINGTON, 2002).

Vários são os problemas relativos às dietas utilizadas para a produção de monossexo que resulta em decréscimo na taxa de sobrevivência, crescimento e reversão sexual das larvas (PANDIAN; SHEELA, 1995), tais como a separação dos ingredientes que ocorre em dietas fareladas, acarretando seletividade por parte das larvas (MEURER et al., 2003).

Segundo Yúfera et al. (1999), para que o uso de dietas inertes seja efetivo, é necessário que sejam ingeridas, digeridas e assimiladas desde os primeiros dias de vida. Para tanto, a microencapsulação das dietas surge como alternativa, possibilitando a utilização de ingredientes de melhor qualidade nutricional, proporcionando melhor nutrição para as larvas e diminuição a lixiviação de nutrientes.

A microencapsulação é um meio de proteger, separar e recobrir materiais em escala microscópica (ALVIM, 2005), possibilitando a liberação de substâncias atrativas e conservando os nutrientes (YÚFERA et al., 2003). As dietas microencapsuladas são mais eficazes em comparação às dietas microaglutinadas ou fareladas como transportadores de substâncias com importância biológica como hormônio estradiol (YÚFERA et al., 2003) e oxitetraciclina (TEMPLE; LANGDON, 2009). A grande vantagem é a menor taxa de lixiviação dos compostos, assegurando melhores respostas aos animais reduzindo a possibilidade de contaminações às pessoas que manipulam a dieta bem como ao ambiente.

Este trabalho teve como objetivo avaliar o uso de diferentes processamentos tecnológicos de microdietas que assegurem melhor reversão sexual da tilápia do Nilo.

\section{MATERIAL E MÉTODOS}

O trabalho foi realizado no Laboratório de Nutrição de Organismos Aquáticos do Centro de Aquicultura da Universidade Estadual Paulista (CAUNESP), no Campus de Jaboticabal.

Foram utilizadas 4000 larvas de $O$. niloticus com cinco dias de idade, que apresentavam 8,31 \pm $0,14 \mathrm{~mm}$ de comprimento total e 4,59 $\pm 0,50 \mathrm{mg}$ de peso úmido. Estas foram acondicionadas em 20 aquários com 50 litros de água, aeração constante e renovação da água de aproximadamente 20 vezes ao dia.

Todas as dietas foram elaboradas com ingredientes de boa qualidade nutricional, respeitando as particularidades de cada processo, como as exigências de umidade, granulometria e, para as dietas microencapsuladas, a concentração de sólidos totais, que é o fator imprescindível para sua produção. As dietas continham $39,67 \pm 4,29 \%$ de proteína bruta e $4655,4 \pm 242,08 \mathrm{kcal} . \mathrm{kg}^{-1}$ de energia bruta com $60 \mathrm{mg}$ de 17 - $\alpha$-metiltestosterona. $\mathrm{kg}^{-1}$ de dieta.

As microdietas foram confeccionadas com granulometria de $500 \mu \mathrm{m}$, adequada para a fase de reversão sexual, denominadas de: FSH - Farelada sem hormônio (controle); e as dietas com hormônio masculinizante (60 mg de 17- $\alpha$-metiltestosterona) FCH - Farelada; MAH - Microaglutinada; SSE Microencapsulada spray dried sem material encapsulante; SCE - Microencapsulada spray dried, 66,7\% de material encapsulante e $33,3 \%$ de dieta (Tabela 1). 
As dietas microencapsuladas (SSE e SCE) foram desenvolvidas no Laboratório de Controle de Qualidade da Faculdade de Engenharia de Alimentos da Universidade de Campinas - SP (UNICAMP). A dieta microencapsulada (SCE) foi constituída de 66,7 de material de encapsulante composto de gelatina e goma arábica na proporção de 1:1 (material encapsulante), e de material de recheio (dieta SSE), técnica descrito por ALVIM (2005).

Tabela 1. Formulação e composição das micro-dietas experimentais.

\begin{tabular}{|c|c|c|c|c|c|}
\hline \multirow{2}{*}{ Ingredientes } & \multicolumn{5}{|c|}{$\operatorname{Dietas}(\%)$} \\
\hline & FSH & FCH & МАН & SSE & $\mathbf{S C E}^{\mathrm{a}}$ \\
\hline Silagem de tilápia inteira ${ }^{\mathrm{b}}$ & - & - & 20,0 & 25,0 & 8,6 \\
\hline Hidrolisado protéico de peixe ${ }^{c}$ & - & - & - & 5,0 & 1,7 \\
\hline Farinha de peixe ${ }^{\mathrm{d}}$ & 27,2 & 27,2 & - & - & - \\
\hline Farinha de sangue desidratado & - & - & 5,0 & - & - \\
\hline Ovo integral desidratado & - & - & 10,0 & 15,1 & 5,2 \\
\hline Clara desidratada de ovo & - & - & - & 10,0 & 3,3 \\
\hline Farelo de soja & 37,1 & 37,1 & 26,6 & - & - \\
\hline Levedura desidratada & 5,0 & 5,0 & 17,9 & 20 & 6,6 \\
\hline Amido de arroz & - & - & 8,0 & 18,1 & 6,0 \\
\hline Gérmen de trigo & - & - & 10,0 & - & - \\
\hline Farelo de trigo & 5,0 & 5,0 & - & - & - \\
\hline Milho moído & 16,2 & 16,2 & - & - & - \\
\hline Óleo de soja & 8,0 & 8,0 & 1,0 & 3,3 & 1,2 \\
\hline Suplemento mineral e vitamínico* & 1,5 & 1,5 & 1,5 & 2,5 & 0,8 \\
\hline Gelatina (material encapsulante) & - & - & - & - & 33,3 \\
\hline Goma arábica (material encapsulante) & - & - & - & - & 33,3 \\
\hline 17- $\alpha$-metiltestosterona $\left(\mathrm{mg} \cdot \mathrm{kg}^{-1}\right)$ & 0 & 60 & 60 & 60 & 60 \\
\hline \multicolumn{6}{|l|}{ Composição Calculada ** } \\
\hline Proteína bruta (\%) & 36,6 & 36,6 & 38,0 & 40,3 & 40,8 \\
\hline Extrato etéreo $(\%)$ & 10,0 & 10,0 & 10,0 & 15,0 & 6,0 \\
\hline Energia bruta $\left(\right.$ Kcal. $\left.\mathrm{kg}^{-1}\right)$ & 4475,3 & 4475,3 & 4500,5 & 4832,9 & 4993,1 \\
\hline
\end{tabular}

FSH - Farelada sem hormônio (controle); FCH - Farelada com hormônio; MAH - Microaglutinada com hormônio; SSE microencapsulada spray dried com hormônio sem material encapsulante; SCE - microencapsulada spray dried com hormônio e 66,7\% de material encapsulante e 33,3\% de dieta. (-) Não apresenta este componente.

*Composição do suplemento mineral e vitamínico: Ferro 15000mg, Cobre 5000 mg, Iodo 500 mg, Manganês 17000 mg, Zinco $12000 \mathrm{mg}$, Selênio $70 \mathrm{mg}$, veículo $1000 \mathrm{~g}$, Vitamina A 12000 UI, Vitamina $\mathrm{D}_{3} 1500$ UI, Vitamina E $50 \mathrm{mg}$, Vitamina K $4 \mathrm{mg}$, Vitamina $B_{12} 7 \mathrm{mg}$, Vitamina $B_{2} 7 \mathrm{mg}$, ácido Pantotênico $60 \mathrm{mg}$, ác. Nicotínico $120 \mathrm{mg}$, cloreto de colina $600 \mathrm{mg}$, metionina $700 \mathrm{mg}$, antioxidante $500 \mathrm{mg}$, veículo $1000 \mathrm{~g}$.

*** Composição calculada com base nas análises dos ingredientes, segundo A.O.A.C. (2000).

${ }^{a}$ Valores de proteína calculado descontando os valores de proteína do material de encapsulante (gelatina e goma arábica).

${ }^{\mathrm{b}}$ Composição: $33,02 \%$ matéria seca, 33,55\% proteína bruta, 25,61\% extrato etério, $4926,43 \mathrm{kcal} . \mathrm{kg}^{-1}$ energia bruta.

${ }^{c}$ Composição: $1,08 \%$ matéria seca, $2,7 \%$ proteína bruta, 44,15 kcal.kg ${ }^{-1}$ energia bruta.

${ }^{d}$ Composição: $95,42 \%$ matéria seca, $60,16 \%$ proteína bruta, 5,84\% extrato etério, $4415,36 \mathrm{kcal} . \mathrm{kg}^{-1}$ energia bruta.

A primeira fase experimental foi de 30 dias, correspondendo à fase de reversão sexual, na qual as larvas de tilápia foram alimentadas seis vezes ao dia "ad libitum". Na segunda fase experimental, todos os 
peixes remanescentes da fase de reversão sexual foram alimentados duas vezes ao dia, "ad libitum", com a dieta FSH passada pelo processo de peletização, por um período de 55 dias.

A segunda fase experimental, de 55 dias, após o período de reversão sexual, foi realizada para permitir o crescimento dos juvenis, possibilitando a retirada das gônadas para verificação da eficiência do tratamento hormonal, pois segundo CARVALHO; FORESTI (1996), antes de 68 dias de vida, as gônadas de tilápia do Nilo apresentam-se indiferenciadas.

A avaliação do crescimento foi realizada no início do experimento $\left(1^{\circ}\right.$ dia $)$, no $15^{\circ}$ e no $30^{\circ}$ dia da fase de reversão sexual. Em cada biometria foram coletados $10 \%$ do total de indivíduos do tratamento para avaliação do peso e do comprimento total. Ao final deste período foi verificada a sobrevivência em cada parcela experimental.

Foram avaliados os parâmetros de peso final, comprimento padrão final, ganho em peso (GP = peso final $[\mathrm{mg}]$ - peso inicial $[\mathrm{mg}])$, Ganho em comprimento $(\mathrm{GC}=$ comprimento final $[\mathrm{cm}]-$ comprimento inicial $[\mathrm{cm}])$, Taxa de crescimento específico $\left(\%\right.$ dia $\left.^{-1}\right)(\mathrm{TCE}=((\ln$ peso final $[\mathrm{mg}]-\ln$ peso inicial [mg]) * 100)/tempo $)$ e sobrevivência $\left(\mathrm{S}=\left(\mathrm{n}^{\mathrm{o}}\right.\right.$ final de larvas *100)/ $\left(\mathrm{n}^{\mathrm{o}}\right.$ inicial de larvas $-\mathrm{n}^{\mathrm{o}}$ de larvas amostradas )

O fator de condição $(K)$ foi calculado pelo método alométrico, a partir da expressão $K=W / L^{b}$, na qual W representa o peso e L, o comprimento padrão dos indivíduos. Para estimar o valor do coeficiente $b$, ajustou-se uma única equação de relação peso/comprimento $\left(\mathrm{W}=\mathrm{aL}^{\mathrm{b}}\right)$, a partir do conjunto de todos os indivíduos coletados, conforme metodologia proposta por VAZZOLER (1996). Para avaliar a uniformidade de peso, foi utilizada uma adaptação da equação proposta por FURUYA et al. (1998): U = (N/N1) x 100, onde: $\mathrm{U}=$ uniformidade (\%); $\mathrm{N}=$ número de animais no tanque; $\mathrm{N} 1$ = número total de animais com peso ou comprimento $20 \%$ superior ou inferior à média do peso vivo, em cada unidade experimental.

Para a análise da efetividade da reversão sexual, após 55 dias do tratamento hormonal, os peixes foram separados em quatro classes de tamanho (maiores de $71 \mathrm{~mm}$; entre 70 a $51 \mathrm{~mm}$; entre 50 a $31 \mathrm{~mm}$ e menores de $30 \mathrm{~mm}$ ). Posteriormente realizou-se o exame das gônadas pelo método do acetato de carmim (WASSERMAN; AFONSO, 2002). O critério utilizado para análise das gônadas foi: macho - presença de cistos de espermatogônia/espermátides e ausência de ovócitos; fêmea - presença de ovócitos e ausência de cistos de espermatogônia/espermátides; intersexuais - presença de cistos de espermatogônia/espermátides e ovócitos; gônadas estéreis ou indefinidas - ausência de cistos de espermatogônia/espermátides ou ovócitos.

Os resultados foram analisados em delineamento inteiramente casualisado (DIC), com cinco tratamentos e quatro repetições. Os resultados de sobrevivência e de taxa de reversão sexual sofreram transformação em arcsen $\sqrt{x / 100}$. O crescimento em peso foi analisado em DIC, em esquema de parcelas subdivididas, tendo como tratamento principal as dietas (FSH, FCH, MAH, SSE e SCE) e como tratamento secundário, os períodos de avaliação biométrica (1, 15 e 30 dias), com quatro repetições. As análises de variância (ANOVA) e as médias foram comparadas pelo testes de Tukey, pelo programa Statistical Analysis System (SAS Intitule Inc., version 6.12, 1999).

\section{RESULTADOS E DISCUSSÃO}

Durante o experimento, os valores médios de temperatura, condutividade, oxigênio dissolvido e pH foram de $29^{\circ} \mathrm{C}, 190,5 \pm 1,5 \mu \mathrm{S} . \mathrm{cm}^{-1}, 5,6 \pm 0,42 \mathrm{mg} . \mathrm{L}^{-1}$ e 7,4 \pm 0,1, respectivamente. Os parâmetros limnológicos mantiveram-se estáveis durante todo o período experimental, dentro de amplitudes consideradas ótimas para a criação da maioria das espécies tropicais de peixes, de acordo com Boyd (1990). 
A homogeneidade inicial do lote foi constatada, pois não foi observada diferença estatística entre os valores de peso e comprimento total das larvas. Após 15 dias, as larvas alimentadas com a dieta FSH, apresentaram crescimento superior às larvas alimentadas com as dietas contendo hormônio (Figura 1). Apesar de vários estudos demonstrarem o efeito do esteróide como promotor de crescimento (DIASKOBERSTEIN et al., 2007; OSTROWSKI ;; GARLING., 1987) até esta fase não foi observado o efeito anabolizante do hormônio masculinizante. Por outro lado, Simone (1990) observou que a administração de dieta com o hormônio 17- $\alpha$-metiltestosterona acarretou em diminuição do crescimento em bagre do canal (Ictalurus punctatus).

Figura 1. Crescimento em peso de tilápia do Nilo alimentadas com microdietas durante a fase de reversão sexual. FSH - Farelada sem hormônio (controle); FCH - Farelada com hormônio; MAH Microaglutinada com hormônio; SSE - microencapsulada spray dryer com hormônio sem material encapsulante; SCE microencapsulada spray dryer com hormônio e $66,7 \%$ de material encapsulante e 33,3\% de dieta.

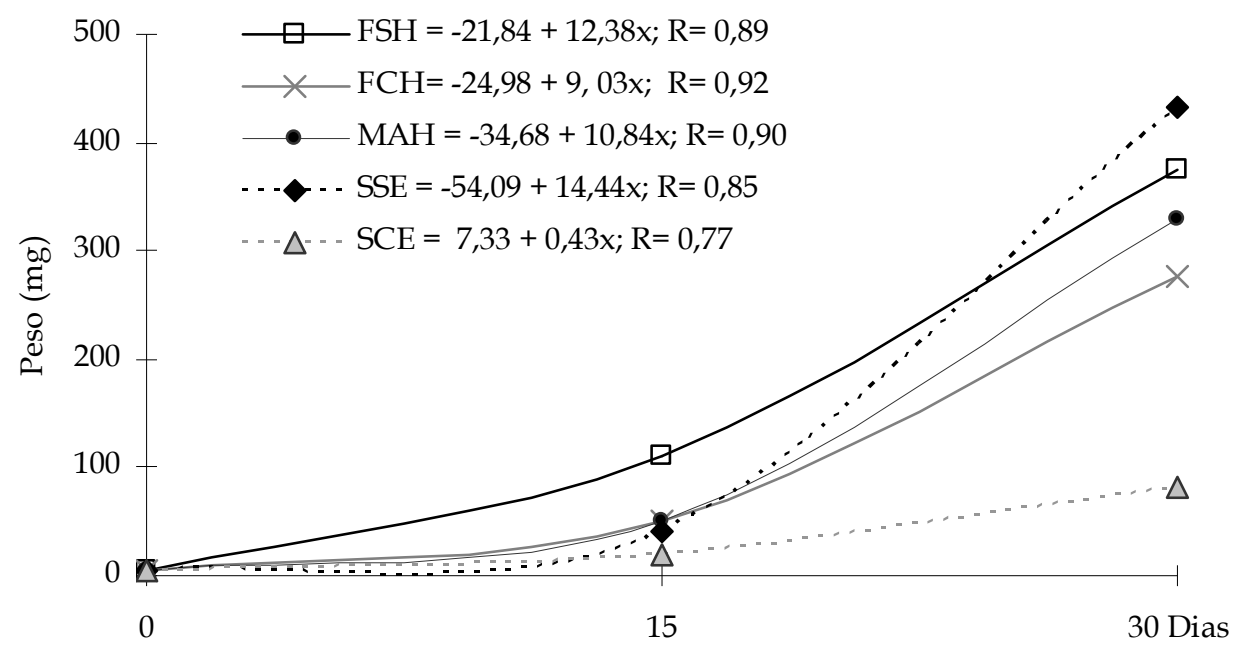

O menor crescimento dos peixes alimentados com a dieta SCE durante o período de reversão sexual pode ser resposta à qualidade nutricional da dieta, que apresentava grande quantidade de material encapsulante. Por outro lado, os peixes alimentados com a dieta SSE apresentaram rápido crescimento nos últimos 15 dias, o que reforça a necessidade de melhora no processo tecnológico de fabricação da dieta, uma vez que a formulação utilizada foi à mesma. Os diferentes processamentos tecnológicos de fabricação das microdietas refletiram em variações nos parâmetros de crescimento (Figura 1, Tabela 2). Ressalta-se que a tilápia do Nilo geralmente não apresenta problemas na ingestão e aproveitamento de dietas na sua fase inicial de alimentação (TACHIBANA et al., 2008), no entanto, a administração da dieta SCE interferiu negativamente na sua eficiência no ganho em peso. O processamento aplicado à dieta (peletização ou extrusão) pode interferir no desempenho de larvas de peixes, como já demonstrado para a tilápia do Nilo por Meurer et al. (2003), Hayashi et al. (1999) e Furuya et al. (1998), como para outras espécie de peixes (RODRIGUES; FERNANDES, 2006; TAKAHASHI et al., 2010).

A fim de obter altas taxas de sobrevivência e crescimento das larvas, a dieta precisa atender as exigências como atratividade, palatabilidade e digestibilidade (TAKAHASHI et al., 2010). Como principal característica física, apresentar alta estabilidade na água e flutuabilidade (BEGSON, 1993). Segundo Alvim (2005) a técnica de microencapsulação empregada na aquicultura visa reduzir a perda por solubilidade de nutrientes das dietas artificiais para a água, diminuindo a poluição e garantindo a qualidade do alimento a ser ingerido. 
A sobrevivência (Tabela 2) das larvas alimentadas com a dieta FCH e MAH foi superior às observadas com as dietas microencapsuladas (SSE e SCE). O uso de hormônio masculinizante não foi o fator responsável pela diferenças na sobrevivência, pois o hormônio estava presente nas mesmas proporções em todas as dietas. Porém, evidencia que o processamento das dietas proporcionou grande influência na sobrevivência. Meurer et al. (2003) observaram altas taxas de sobrevivência de larvas de tilápia alimentadas com dietas fareladas, em comparação com as de dietas pastosas e micropeletizadas. Os valores de sobrevivência encontrados neste estudo estão de acordo com os observados para tilápia do Nilo na fase de reversão sexual (NEUMANN et al., 2009; DIAS-KOBERSTEIN et al., 2007).

$O$ fator de condição apresentou diferença significativa $(p<0,05)$ entre as diferentes dietas fornecidas (Tabela 2), sendo que as larvas de tilápia alimentadas com as dietas MAH e SSE apresentaram os melhores resultados, indicando que estes apresentam melhor conformação corporal (relação peso: comprimento) refletindo em índice de bem estar segundo Portela et al. (2000).

Tabela 2. Médias \pm desvio padrão dos parâmetros de desempenho produtivo de larvas de tilápia do Nilo (Orechromis niloticus) alimentadas com diferentes microdietas durante a fase de reversão sexual.

\begin{tabular}{lccccc}
\hline & FSH & FCH & MAH & SSE & SCE \\
\hline PF & $376,11 \pm 9,2 \mathrm{a}$ & $275,56 \pm 9,6 \mathrm{ab}$ & $329,81 \pm 9,1 \mathrm{a}$ & $423,44 \pm 15,6 \mathrm{a}$ & $80,96 \pm 1,4 \mathrm{~b}$ \\
$\mathbf{C P F}$ & $2,61 \pm 0,1 \mathrm{a}$ & $2,45 \pm 0,2 \mathrm{a}$ & $2,59 \pm 0,1 \mathrm{a}$ & $2,85 \pm 0,28 \mathrm{a}$ & $1,73 \pm 0,1 \mathrm{~b}$ \\
$\mathbf{G P}$ & $371,32 \pm 93,1 \mathrm{a}$ & $271,01 \pm 87,3 \mathrm{a}$ & $325,26 \pm 77,7 \mathrm{a}$ & $427,34 \pm 156,7 \mathrm{a}$ & $76,78 \pm 19,3 \mathrm{~b}$ \\
GC & $1,78 \pm 0,1 \mathrm{a}$ & $1,67 \pm 0,1 \mathrm{a}$ & $1,72 \pm 0,1 \mathrm{a}$ & $2,08 \pm 0,3 \mathrm{a}$ & $0,87 \pm 0,2 \mathrm{~b}$ \\
TCE & $14,54 \pm 1,3 \mathrm{a}$ & $13,58 \pm 0,8 \mathrm{a}$ & $14,24 \pm 0,6 \mathrm{a}$ & $14,67 \pm 1,5 \mathrm{a}$ & $9,85 \pm 1,0 \mathrm{~b}$ \\
$\mathbf{K}$ & $5,90 \pm 0,3 \mathrm{~b}$ & $3,33 \pm 0,6 \mathrm{~b}$ & $13,80 \pm 2,6 \mathrm{a}$ & $9,10 \pm 1,8 \mathrm{a}$ & $3,40 \pm 0,5 \mathrm{~b}$ \\
$\mathbf{U}$ & $5,10 \pm 4,6 \mathrm{~d}$ & $15,12 \pm 4,8 \mathrm{c}$ & $25,11 \pm 4,5 \mathrm{~b}$ & $40,02 \pm 7,8 \mathrm{a}$ & $27,08 \pm 0,7 \mathrm{~b}$ \\
$\mathbf{S}$ & $76,20 \pm 0,9 \mathrm{~b}$ & $96,70 \pm 2,2 \mathrm{a}$ & $89,50 \pm 6,8 \mathrm{a}$ & $76,61 \pm 3,3 \mathrm{~b}$ & $64,03 \pm 1,7 \mathrm{~b}$ \\
\hline
\end{tabular}

Valores seguidos das mesmas letras iguais na linha demonstram que não houve diferença significativa a $5 \%$ de probabilidade pelo teste de Tukey.

$P F$ - Peso final (mg); CPF - Comprimento padrão final (cm); GP - Ganho em peso ( $\mathrm{mg}$ ), GC - Ganho em comprimento $(\mathrm{cm}) ;$ TCE - Taxa de crescimento específico $\left(\%\right.$ dia $\left.^{-1}\right) ; K$ - Fator de condição $\left(k .10^{-3}\right) ; U$ - Uniformidade de peso $(\%), S$ Sobrevivência (\%)

FSH - Farelada sem hormônio (controle); FCH - Farelada com hormônio; MAH - Microaglutinada com hormônio; SSE - microencapsulada spray dryer com hormônio sem material encapsulante; SCE - microencapsulada spray dryer com hormônio e $66,7 \%$ de material encapsulante e $33,3 \%$ de dieta.

Os peixes alimentados com a dieta FSH apresentaram uma proporção sexual de 43,25\% de machos e 56,75\% de fêmeas, uma relação próxima de 1:1. Em nenhum dos tratamentos foram observados animais intersexo. As tilápias alimentadas com a dieta SCE apresentaram valores de reversão similar entre as demais microedietas (SSE e MAH). No entanto, diferiram do tratamento farelada com hormônio (FCH) que resultou em valor médio de 76,20\% (Figura 2). Segundo Popma e Lovshin, (1996), o percentual de $80 \%$ de machos após o tratamento hormonal é considerado baixo. Desta forma pode-se observar que a reversão sexual foi mais eficaz nos tratamentos SCE, SSE e MAH do que o método comumente aplicado nas pisciculturas. Esse fato reforça a idéia de melhora no processo tecnológico de fabricação de rações. 
Figura 2. Porcentagem de machos de tilápia do Nilo alimentadas com microdietas durante a fase de reversão sexual. FSH - Farelada sem hormônio (controle); FCH - Farelada com hormônio; MAH Microaglutinada com hormônio; SSE - microencapsulada spray dryer com hormônio sem material encapsulante; SCE - microencapsulada spray dryer com hormônio e 66,7\% de material encapsulante e $33,3 \%$ de dieta.

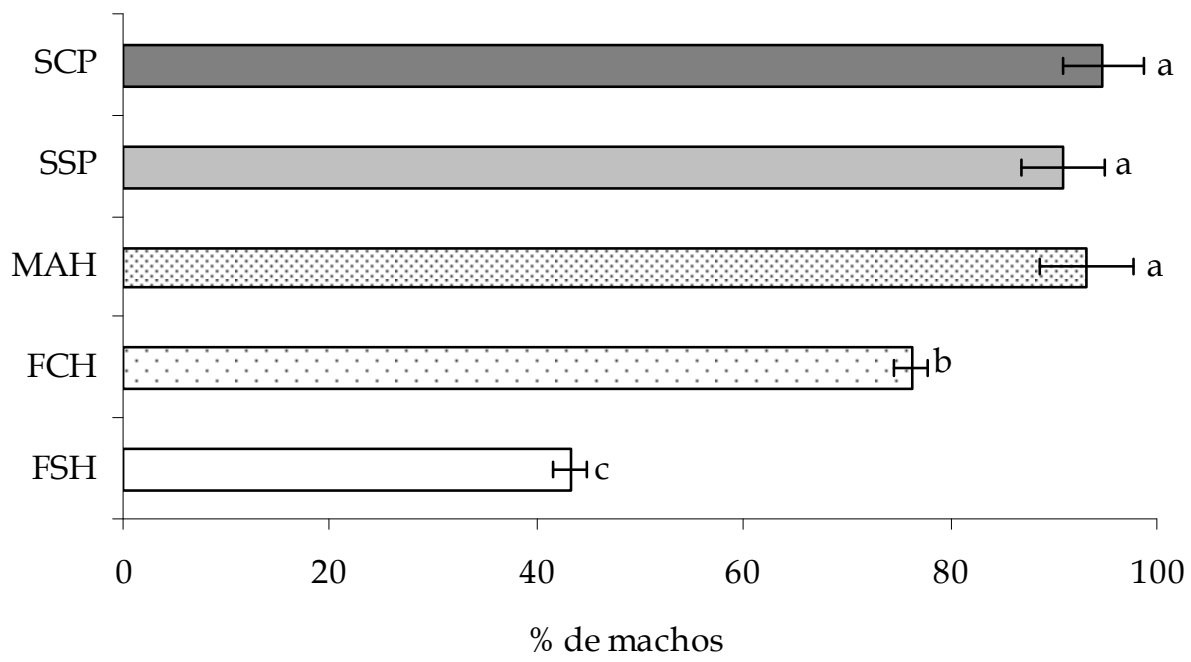

O melhor resultado de uniformidade do lote foi observado pelos peixes alimentados com a dieta SSE (Tabela 2), podendo atribuir esta resposta a maior estabilidade da dieta na água devido ao material encapsulante endógeno (amido da dieta) (ALVIM, 2005), o que favorece a oferta de alimento de qualidade para os peixes independente da velocidade de alimentação (MEURER et al., 2005).

Embora a análise de variância não tenha revelado efeitos significativos na distribuição de peixes por classe de tamanho (Figura 3), observou-se que, a dieta SSE apresentou o maior número de peixes acima de $71 \mathrm{~mm}$, não possuindo peixes abaixo de $30 \mathrm{~mm}$ de comprimento, confirmando sua homogeneidade ao final da fase de reversão sexual (Tabela 2), tornando se o grupo mais homogêneo.

Figura 3. Distribuição percentual dos peixes por classe de tamanho, após 55 dias do período de reversão sexual. FSH - Farelada sem hormônio (controle); FCH - Farelada com hormônio; MAH Microaglutinada com hormônio; SSE - microencapsulada spray dryer com hormônio sem material encapsulante; SCE - microencapsulada spray dryer com hormônio e 66,7\% de material encapsulante e $33,3 \%$ de dieta.

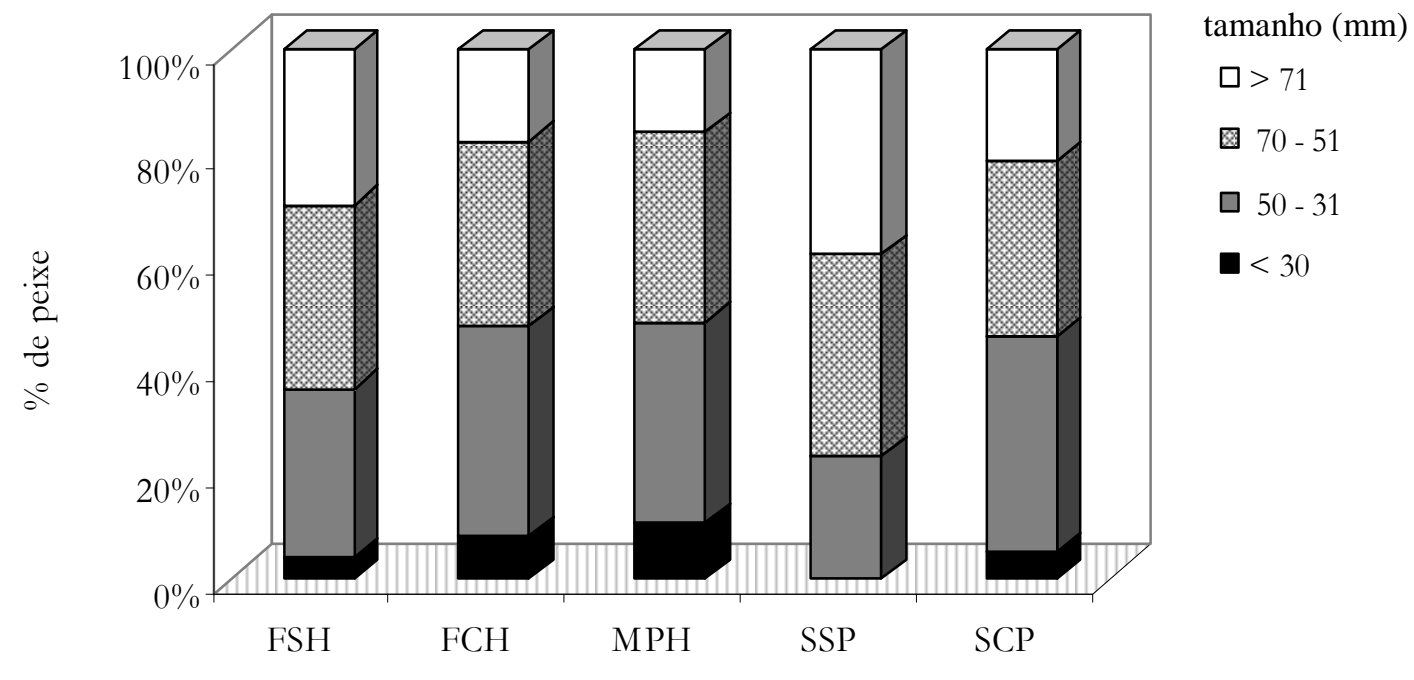


Alguns estudos foram desenvolvidos com microdietas para larvas de peixe (YÚFERA et al., 2002; KOVALENCO et al., 2002) demonstrando os avanços das dietas microencapsuladas para obtenção de propriedades desejadas como estabilidade, aceitação e desintegração no estômago das larvas. A qualidade das técnicas de microencapsulação que são recomendadas também para englobar diferentes substâncias que precisam ser vinculadas pela dieta, como vitaminas, hormônios e aminoácidos (YÚFERA et al., 2003), foram confirmados neste estudo para produção de peixes sexualmente revertidos com desempenho e homogeneidade do lote que viabilizam a próxima fase de cultivo.

Conclui-se que as microdieta MAH e SSE são as mais eficazes a serem utilizadas na fase de reversão sexual. Devido aos altos índices de reversão sexual observados pelo tratamento SCE, maiores estudos devem ser realizados para que promova maior eficiência alimentar.

\section{AGRADECIMENTOS}

Ao Prof. Dr. Carlos Grosso e sua equipe da Universidade Estadual de Campinas UNICAMP, Faculdade de Engenharia de Alimentos, Departamento de Alimentos e Nutrição pela confecção das dietas microencapsuladas experimentais.

\section{REFERÊNCIAS}

ALVIM, I.D. Produção e caracterização de microparticulas obtidas por spray drying e coacervação complexa e seu uso para alimentação de larvas de peixes. Campinas. 76p. 2005. Tese (Doutoramento. Engenharia de Alimentos). Universidade de Campinas.

AOAC - ASSOCIATION OF OFFICIAL ANALYTICAL CHEMISTS.Official Methods of Analysis, 17th edn. Gaithersburg, MD, USA: AOAC Inc., 2000.

BEARDMORE, J.A.; MAIR, G.C.; LEWIS, R.I. Monosex male production in finfish as exemplified by tilapia: applications, problems, and prospects. Aquaculture, Amsterdam, n.197, p.283-301, 2001.

BEGSON, D. A comprehensive program for the evolution of artificial diets. Journal of the World Aquaculture Society, Baton Rouge, v.24, p.199-210, 1993.

BOYD, C.E. Water quality in ponds for aquaculture. Auburn: Auburn University, 1990. 482p.

CARVALHO, E.D.; FORESTI, F. Reversão sexual em tilápia do Nilo Oreochromis niloticus Trewavas, 1983, induzida por 17- $\alpha$-metiltestosterona: proporção de sexo e histologia das gônadas. Revista Brasileira de Biologia, São Carlos, v.2, p. 249-262, 1996.

DIAS-KOBERSTEIN, T.C.R. et al. Reversão sexual de larvas de tilápia do Nilo (Oreochromis niloticus) por meio de banhos de imersão em diferentes dosagens hormonais. Revista Acadêmica, Curitiba, v.5, n.4, p. 391-395, 2007.

FURUYA, W.M.et al. Dietas peletizada e extrusada para machos revertidos de tilápia do Nilo (Oreochromis niloticus) na fase de terminação. Ciência Rural, Santa Maria, v.28, n.3, p. 483-487, 1998.

GREEN, B.W.; TEICHERT-CODDINGTON, D.R. Human food safety and environmental assessment of the use 17- $\alpha$-methyltestosterone to produce male tilapia in the United States. . Journal of the World Aquaculture Society, Baton Rouge, v.31, n. 3, p. 337-357, 2002.

HAYASHI, C.et al. Uso de diferentes graus de moagem dos ingredientes em dietas para a tilápia-do-Nilo (Oreochromis niloticus L.) na fase de crescimento. Acta Scientiarum, Maringá, v.21, n.3, p. 733-737, 1999. 
KOVALENCO, E.E. et al. A successful microbound diet for the larval culture of freshwater prawn. Macrobrachium rosenbergii. Aquaculture, Amsterdam, 210: 385-395, 2002.

MAINARDES-PINTO, C.S.R.et al. Masculinização da tilápia do Nilo, Oreochromis niloticus, utilizando diferentes rações e diferentes doses de $17 \alpha$-Metiltestosterona. Revista Brasileira de Zootecnia, Viçosa, v.29, n.3, p. 654-659, 2000.

MEURER, F.et al. Grau de moagem dos alimentos em rações para a tilápia do Nilo (Oreochromis niloticus) durante o período de reversão sexual. Acta Scientiarum. Animal Sciences, Maringá, v. 27, n.1, p. 81-85, 2005.

MEURER, F.; HAYASHI, C.; BOSCOLO, W.R. Influência do processamento da ração no desempenho e sobrevivência da tilápia do Nilo durante a reversão sexual. Revista Brasileira de Zootecnia, Viçosa, v.32, n.2, p. 262-267, 2003.

NEUMANN, E.; DIAS KOBERSTEIN, T.C.; BRAGA, F.M.S. Desempenho de três linhagens de tilápia submetidas ao tratamento com 17- $\alpha$-metiltestosterona em condições ambientais não controladas. Revista Brasileira de Zootecnia, Viçosa, v.38, n.6, p. 973-979, 2009.

OSTROWSKI, A.C.; GARLING, D.L. JR. Effect of $17 \alpha$ methyltestosterone treatment and withdrawal on growth and dietary protein utilization of juveniles rainbow trout fed practical diets varying in protein level. . Journal of the World Aquaculture Society, Baton Rouge, v.18, p. 71-77, 1987.

PANDIAN, T.J.; SHEELA, S.G.Hormonal induction of sex reversal in fish. Aquaculture, Amsterdam, v.138, p. 1-22, 1995.

POPMA, T.J.; LOVSHIN, L. Worldwide prospects for commercial production of tilapia. International Center for Aquaculture and Aquatic Environments. Auburn, Alabam. Research and Development. 1996. 23p. (Series n. 41).

PORTELLA, M.C.; VERANI, J.R.; CESTAROLLI, M.A. Use of live and artificial diets enriched with several fatty acid source to feed Prochilodus scrofa larvae and grow rates. Journal of Aquaculture in the Tropics, Calcutá - Índia, v.15, n.1, p. 45-58, 2000.

RODRIGUES, L.A.; FERNANDES, J.B.K. Influência do processamento da dieta no desempenho produtivo do acará bandeira (Pterophyllum scalare). Acta Scientiarum, Maringá, v.28, n.1, p. 113-119, 2006.

SIMONE, D.A. The effects of the synthetic steroid 17-alfa-methyltestosterone on the growth and organ morphology of the channel catfish (Ictalurus punctatus). Aquaculture, Amsterdam, v.84, n. 81-93, 1990.

SAS - STATICAL ANALISYS SYSTEM. Institute, SAS/STAT SAS/STAT. User's guide: statistic: Versão 6.08.4. ed. Cary.

TACHIBANA, L.et al. Densidade de estocagem de pós-larvas de tilápia-do-Nilo (Oreochromis niloticus) durante a fase de reversão sexual. Boletim do Instituto de Pesca, São Paulo, v. 34, n.4, p. 483-488, 2008.

TAKAHASHI, L.S.et al. Efeito do tipo de alimento no desempenho produtivo de juvenis de Acarábandeira (Pterophyllum scalare). Boletim do Instituto de Pesca, São Paulo, v.36, n.1, p. 1-8, 2010.

TEMPLE, E.; LANGDON, C. Delivering oxytetracycline to first-feeding zebrafish, Danio rerio (Hamilton), and goby, Asterropteryx semipunctata Rüppell, larvae using lipid spray beads. Journal of Fish Diseases, Auburn, v.32, p. 279-292, 2009.

VAZZOLER, A. Biologia da reprodução de peixes teleósteos: teoria e prática. Maringá:EDUEM, 1996. 169p. 
WASSERMAN, G.J.; AFONSO, L.O.B. Sex reversal in Nile tilápia (Oreochromis niloticus, Linnaeus) by androgen immersion. Aquaculture Research, Malden, v. 34, p.65-71, 2002.

YÚFERA, M.; PASCULA, E.; FERNÁNDEZ-DIÁZ, C.. A highly efficient microencapsulated food for rearing early larvae of marine fish. Aquaculture. Amsterdam, n.177, p. 249-256, 1999.

YÚFERA M.et al. Free amino acid leaching from a protein-walled microencapsulated diet for fish larvae. Aquaculture, Amsterdam, v.214, p. 273-287, 2002.

YÚFERA M.et al. Delivering bioactive compounds to fish larvae using microencapsulated diets.

Aquaculture, Amsterdam, v.277 p. 277-291, 2003. 